La revue La revue pour l'histoire du CNRS

POUR LHISTORE DU CNRS $\quad 18 \mid 2007$

Voyages collectifs en géographie

Les « sciences morales » : de la gloire à l'oubli?

Savoirs et politique en Europe au XIX ${ }^{\mathrm{e}}$ siècle

Julien Vincent

(2) OpenEdition

Journals

Édition électronique

URL : https://journals.openedition.org/histoire-cnrs/4551

DOI : 10.4000/histoire-cnrs.4551

ISSN : 1955-2408

Éditeur

CNRS Éditions

Édition imprimée

Date de publication : 3 octobre 2007

ISBN : 978-2-271-06559-9

ISSN : $1298-9800$

Référence électronique

Julien Vincent, « Les « sciences morales » : de la gloire à l'oubli ? », La revue pour l'histoire du CNRS [En ligne], 18 | 2007, mis en ligne le 03 octobre 2009, consulté le 20 mai 2021. URL : http://

journals.openedition.org/histoire-cnrs/4551; DOI : https://doi.org/10.4000/histoire-cnrs.4551

Ce document a été généré automatiquement le 20 mai 2021

Comité pour I'histoire du CNRS 


\title{
Les « sciences morales » : de la gloire à l'oubli ?
}

\author{
Savoirs et politique en Europe au XIX ${ }^{\mathrm{e}}$ siècle
}

Julien Vincent

1 Les «sciences morales » ne sont pas le poussiéreux mélange de science et de morale qu'on oppose trop souvent aux «sciences humaines» ou aux «sciences sociales». Ce n'est qu'au cours du $\mathrm{XX}^{\mathrm{e}}$ siècle que ces dernières s'imposent au sein des institutions officielles d'enseignement et de recherche. Mais au XIXe siècle leur légitimité était loin d'être acquise face au modèle rival des «sciences morales et politiques". Si les académies qui arborent ce nom aujourd'hui, dans différents pays d'Europe, remplissent un rôle de consécration plutôt que d'invention et d'innovation en sciences humaines et sociales, ce ne fut pas toujours le cas. Elles étaient, au XIX ${ }^{e}$ siècle, au centre d'un effort soutenu et international pour organiser la recherche autant que ses applications sociales et politiques ${ }^{1}$.

2 Continuant l'œuvre des influentes académies du XVIII ${ }^{\mathrm{e}}$ siècle, dont les prix convoités stimulaient les travaux des écrivains des Lumières, elles sont le chaînon manquant qui permet de relier ces dernières aux universités modernes et aux organismes de recherche du XX ${ }^{\mathrm{e}}$ siècle, à l'image de la Caisse des recherches scientifiques née en 1901, ancêtre du CNRS.

3 L'idée de «sciences morales » ne va pourtant pas de soi. Définies négativement comme ces sciences qui ne sont pas physiques ou naturelles, elles sont synonymes, au XIX ${ }^{\mathrm{e}}$ siècle, de «sciences mentales " plutôt que d'éthique (même si elles incluent en général la philosophie morale et politique). Au-delà de cet accord minimal, ni les frontières ni le contenu et l'architecture interne de cet ensemble de disciplines ne fait l'objet d'un consensus. Tout au long du XIX ${ }^{e}$ siècle, la définition des sciences morales ne cessera de poser problème. C'est peut-être cet inachèvement et le caractère instable et disputé des sciences «non- physiques » qui contribua le mieux à faire émerger une nouvelle organisation des savoirs, spécifique au XIXe siècle. On peut en souligner trois aspects : la volonté de représenter les nouvelles connaissances sur l'homme au sein 
d'institutions officielles; l'espoir que ces connaissances auront des retombées politiques et sociales; enfin l'essor d'une nouvelle économie internationale des savoirs. Un « parlement » des sciences de l'homme et de la société ?

4 Le terme de "sciences morales et politiques» (et ses équivalents dans les langues étrangères : moral and political science, ciencias morales y politicas, scienze morali e politiche, geisteswissenschaften) reflète dès l'origine le projet d'une classification des savoirs anciens et nouveaux sur l'homme et la société afin de les représenter au sein d'institutions officielles. Dans un contexte où la question du suffrage et de la meilleure forme de représentation politique surgit régulièrement dans les débats publics, c'est aussi de la représentation scientifique de la nation que l'on se préoccupe ${ }^{2}$. Comme le note le ministre de l'Intérieur belge, Sylvain Van de Weyer, dans une lettre au roi Léopold Ier justifiant la création d'une nouvelle section vouée aux sciences morales, «l'organisation actuelle de l'Académie royale de sciences et Belles Lettres de Bruxelles n'est plus en harmonie avec les progrès que la science et la littérature ont faits dans notre pays " ${ }^{3}$. Les sciences morales joueront le rôle d'un véritable "parlement » des sciences de l'homme et de la société.

5 Tout commence sous la Révolution française avec la création d'une « classe des sciences morales et politiques » au sein de l'Institut de $1795{ }^{4}$. Si Napoléon Bonaparte interrompt le projet des Thermidoriens dès 1803 , les sciences morales continuent de faire l'objet de multiples fondations tout au long du XIX siècle. Déjà l'Institut national italien de Bologne de 1802, intégré à la jeune République italienne, tout comme la nouvelle Società Pontaiana de Naples de 1808, présidée par Vincenzo Cuoco, avaient comporté une classe des sciences morales et politiques sur le modèle français. Guizot relance la dynamique en fondant l'Académie des sciences morales et politique au sein de l'Institut en 1832, avec l'objectif affiché de reprendre l'œuvre interrompue près de trente ans plus tôt. L'Académie royale de Belgique, refondée en 1832, se dote officiellement d'une "classe des sciences morales et politiques » à partir de 1843. En Angleterre, c'est le Prince Albert (le mari de la reine Victoria) qui, ouvert aux idées libérales par son tuteur Adolphe Quételet et profitant du contexte politique agité sur le continent, impose à William Whewell et à ses collègues la création d'un Tripos de sciences morales à Cambridge en $1848^{5}$. En 1853, une section du même nom est fondée au sein de l'Institut national genevois créé sur le modèle de l'Institut de France par James Fazy, ancien opposant parisien à Louis-Philippe et fondateur du parti radical genevois. En 1857, une Académie royale de sciences morales est fondée à Madrid ${ }^{6}$. En 1861, une Académie des sciences morales et politiques est fondée au sein de la Société royale de Naples; en 1864 une classe du même nom naît au sein de l'Institut royal lombard des sciences et des lettres; et en 1874, au sein de l'Académie nationale et royale des Lincei à Rome.

6 La juste représentation de chaque discipline fait l'objet d'âpres combats. Si l'insertion des domaines traditionnels de la philosophie (métaphysique, logique, philosophie politique et morale) ne pose guère de problème, celle de la psychologie, du droit, de l'histoire, des sciences sociales, de l'économie politique, de la géographie ou de la philologie fait l'objet d'importantes variations d'un pays à l'autre. Les luttes pour la définition d'un corpus disciplinaire et d'une méthode varient aussi fortement en fonction des contextes politiques et des environnements institutionnels. Ainsi l'Académie de Guizot dominée par le philosophe Victor Cousin, chef de l'école éclectique, abandonne les présupposés sensualistes de l'Institut de 1795 qui semblent trop révolutionnaires. Au lieu de commencer la science morale par «l'analyse des 
sensations et des idées ", elle privilégie l'étude de l'âme et de la philosophie morale. De même, alors que les académies continentales ignorent le " conflit des facultés " propre au monde universitaire, les sciences morales anglaises sont intégrées au cursus de Cambridge où la concurrence entre les disciplines revêt des enjeux de pouvoir spécifiques qui conduisent souvent à la revendication d'autonomie, à l'image des historiens et des juristes qui sortent du Tripos de sciences morales en 1867.

7 L'exemple anglais illustre les différentes manières dont les sciences morales disposent $\mathrm{du}$ tissu institutionnel existant. Sur le continent, les académies nationales et provinciales, généralement fondées aux XVII ${ }^{e}$ et XVIII ${ }^{e}$ siècles, s'adaptent bien à un projet qui est non seulement d'offrir un lieu permanent pour accueillir les recherches des meilleurs savants, mais aussi de mobiliser au coup par coup les forces intellectuelles dispersées autour d'un sujet précis (notamment par le moyen de prix), et de diffuser les connaissances les plus « utiles » auprès du public. Mais en Angleterre, où de telles académies n'existent pas, ces fonctions sont séparées ' 7 . La Social Science Association, fondée en 1857 et dissoute en 1886, remplit le rôle de pompe aspirante et refoulante des informations statistiques et des idées politiques reliant le parlement, le monde savant et la société civile ${ }^{8}$. Le Tripos des sciences morales de Cambridge, quant à lui, promeut une recherche de pointe dans diverses disciplines, et s'efforce de les enseigner aux futures élites de la nation, les étudiants des collèges de Cambridge.

Savoirs et politique à l'ère du libéralisme

8 La question de l'enseignement des sciences morales fait l'objet de houleux débats qui en révèlent la portée politique. En Belgique, le projet de Charles Rogier de faire de l'Académie un corps d'enseignants est contré au parlement par Barthélemy du Mortier en 1834, ouvrant une controverse durable qui explique en partie l'adoption tardive de nouveaux statuts en $1845^{9}$. Même à Cambridge, où les sciences morales sont intégrées au cursus universitaire, elles sont réservées, jusqu'en 1860, aux seuls étudiants " gradués » qui ont déjà étudié pendant trois ans les mathématiques ou les humanités. Pourtant les défenseurs des sciences morales, à l'image de Guizot (en France) et de Moyano (en Espagne), sont aussi d'ardents promoteurs de l'instruction publique. Si les nouvelles sciences "non physiques » ont pour mission d'œuvrer au progrès général, elles doivent rester sous le contrôle d'une aristocratie du savoir ${ }^{10}$.

Cette ambivalence n'est pas nouvelle. Déjà en 1795, lorsqu'ils avaient fondé l'Institut, les hommes de Thermidor avaient décidé de publier, à titre posthume et aux frais de la République, l'Esquisse d'un tableau historique des progrès de l'esprit humain de Condorcet. La nouvelle République portait en effet le deuil du mathématicien et philosophe mort en prison en mars 1794 pendant les derniers moments du gouvernement de Robespierre. Dès leur naissance les sciences morales se placent ainsi sous le double signe d'une foi dans le progrès humain et d'une méfiance à l'égard de la démocratie. Parlement des savoirs sur l'homme, les sciences morales seront donc aussi un « Sénat intellectuel de la Nation " ${ }^{11}$. Les fondations de Paris, Naples, Bruxelles, Cambridge, Genève ou Madrid, apparemment si éloignées dans leur géographie comme dans leur chronologie, sont l'expression de cette problématique politique commune à toute l'Europe du XIX siècle.

10 Si la République française a triomphé institutionnellement sous la Révolution, elle n'a pas su se faire aimer et n'est pas parvenue à créer les conditions économiques de sa réussite ${ }^{12}$. La démocratie, horizon indépassable selon Alexis de Tocqueville (membre de l'Académie des sciences morales et politiques à partir de 1838) ne pourra se faire de 
manière satisfaisante sans une adhésion des cours et des corps. Or un retour en arrière semble inenvisageable : les monarchies censitaires d'Europe sont toutes confrontées à la question des libertés religieuses et du statut des églises officielles, de la liberté de la presse, de la propriété ecclésiastique ou aristocratique et de l'élargissement du droit de vote. Il faut donc promouvoir, antérieurement à l'instauration d'institutions pleinement démocratiques, un progrès matériel, intellectuel et moral. Samuel Coleridge résume bien cette idée lorsqu'il explique depuis Cambridge, en 1829, que les forces de progrès ne peuvent se réaliser elles-mêmes sans la collaboration des forces de conservation. Jules Lechevalier, éditeur des Archives de sciences morales et politiques, parle des «deux forces de conservation et d'innovation dont l'action réciproque constitue le progrès normal de l'esprit humain ${ }^{13}$. Pour le marquis José de Pidal, ouvrant la séance inaugurale de l'Académie madrilène le 19 septembre 1858, il faudra s'opposer aux « innovations radicales » qui pourraient, par exemple, remettre en cause la propriété privée; il faudra aussi, dit-il, «rechercher constamment ce qui peut améliorer nos lois et nos institutions, détruire les erreurs dangereuses, instruire les classes moins éclairées, leur inspirer le sentiment du devoir, l'amour de l'ordre, le respect de l'autorité. Tout ce qui, en un mot, est susceptible de rendre meilleure la condition de chacun en élevant son caractère moral $»{ }^{14}$. On retrouve, mutatis mutandis, le projet des Girondins français disciples de Condorcet.

11 Un tel projet suppose l'unification d'une opinion publique (aussi désignée par le terme de "sens commun ») souvent traversée par d'importants clivages religieux et culturels. En France, l'Académie de Guizot veut réconcilier la France de l'Ancien Régime et celle de la Révolution ${ }^{15}$. D'autres pays cherchent à refermer des plaies plus anciennes. En Angleterre, l'histoire du Tripos des sciences morales de Cambridge accompagne l'abolition progressive des privilèges des Anglicans et l'entrée des Dissidents qui sont officiellement admis comme étudiants à Oxford et à Cambridge au milieu des années 1850, puis comme enseignants en $1871^{16}$. En Espagne, les sciences morales sont issues des luttes et des révolutions qui opposent libéraux et modérés. Insérées dans la loi sur l'enseignement secondaire et universitaire de Claudio Moyano, elles participent d'une tentative de stabilisation sociale et idéologique suite au retour au pouvoir des modérés. Dans les nouvelles nations la situation est encore différente. Déjà en 1823, dans un contexte de luttes nationales, un Colegio de ciencias morales avait été fondé à Buenos Aires sous l'influence du futur président argentin Bernardo Rivadavia. En Belgique, après 1830, Léopold Ier veut faire de son jeune royaume une nation moderne et industrielle capable de surmonter ses divisions culturelles et linguistiques entre wallons et flamands. Ces derniers obtiennent à partir de 1845 une meilleure représentation au sein de l'Académie de Bruxelles qui est alors chargée de publier une Biographie nationale. À Naples, où Garibaldi entre en septembre 1860, la réforme de l'Académie royale répond à la libéralisation du régime, à la destruction de l'Europe du Congrès de Vienne que Napoléon III cherche à accélérer, et à la formation d'un Royaume d'Italie unifiant Italiens du Nord et Italiens du Sud.

12 Tels sont les défis auxquels sont confrontées les sciences morales, qui s'arrogent l'objectif ambitieux de remettre en phase l'évolution des institutions politiques avec celle de la science et des différentes facultés humaines. Il s'agit de rien de moins que de ralentir l'histoire, ou plutôt de réconcilier le temps de la politique avec celui de la science. On permettra ainsi un « rattrapage » des facultés productives, intellectuelles et morales de l'homme et de la société qui permettra de compenser l'accélération démocratique qu'a provoquée la Révolution française. Pourtant vie intellectuelle et vie 
politique obéissent à des temporalités différentes. Les diverses institutions qui se consacrent aux sciences morales en font l'expérience parfois amère. Face à l'urgence des réformes, la science ne va pas assez vite. Faut-il repenser le système pénitentiaire, diminuer le temps de travail des enfants ou des femmes, modifier le statut des associations d'ouvriers, changer les dispositions légales qui encadrent la vente ou la transmission des terres ? Il faudrait, avant de pouvoir régler de tels problèmes, avoir eu le temps de réunir toute l'information nécessaire, de l'avoir absorbée, assimilée et retransmise. Or la politique n'attend pas.

Des circulations internationales

13 Afin de gagner un peu de ce temps de la science toujours trop lent par rapport au temps de la politique, une stratégie possible est de raccourcir l'espace. Vers le milieu du XIX ${ }^{\mathrm{e}}$ siècle et avant la création de l'Association internationale des Académies, en 1901, les sciences morales sont une arène privilégiée de la circulation des travaux d'un pays à l'autre. Du fait de leur enveloppe institutionnelle et des pratiques qu'elle suscite (exposition de bustes, séances solennelles...), elles fonctionnent comme une instance de consécration à la fois nationale et internationale qui œuvre à construire l'idée d'une science « étrangère » en même temps que d'en faciliter l'importation. Que ce soit par la publication d'ouvrages étrangers, par l'organisation de concours promouvant l'analyse comparée de différentes situations nationales ${ }^{17}$, par l'élection de membres étrangers et de membres correspondants en plus de leurs membres permanents nationaux, ou par l'insertion de leurs œuvres dans des bibliographies, les sciences morales contribuent indissociablement à élever des frontières et à les franchir ${ }^{18}$. De telles pratiques sont très réglementées. Ainsi, les mémoires étrangers publiés par l'Académie royale des sciences morales et politiques française, qui doivent obligatoirement être inédits, font l'objet d'un rapport de la section concernée ainsi que d'un vote au bulletin secret ${ }^{19}$. De même, à Cambridge, la constitution de bibliographies officielles à l'usage des étudiants, à partir desquelles les enseignants mettent en dialogue "pensée anglaise ", "pensée française » et " pensée allemande » dans leurs cours, font l'objet de rivalités sans merci entre les membres du Moral Science Board.

Ceci facilite la constitution d'un cadre de référence commun sur des questions aussi diverses que la propriété, la famille ou les rapports entre le corps et l'âme. Les mêmes controverses, les mêmes écoles de pensée semblent se répliquer d'un pays à l'autre. L'Académie madrilène, dans ses points d'accord comme dans ses divisions, est selon un observateur belge « en complète communauté d'idées sur les grandes questions sociales avec l'École française" ${ }^{20}$. Victor Cousin, qui domine l'Académie parisienne, fait connaître à toute l'Europe l'idéalisme allemand et la philosophie écossaise du sens commun en les présentant comme des philosophies «actuelles». En Belgique, Van Meenen, Van de Weyer et le baron de Reiffenberg, véritable "triumvirat de l'éclectisme ", reprennent les problématiques de Cousin et combattent le sensualisme ${ }^{21}$. En face, Adolphe Quételet, promoteur de la "physique sociale» qui défend une approche statistique et déterministe des "phénomènes moraux " (mariages, suicides, crimes), n'a pas moins d'influence internationale. Secrétaire perpétuel de l'Académie bruxelloise de 1834 à sa mort en 1874, on peut dire qu'il est prophète en son pays, à la différence de John Stuart Mill dont les idées empiristes et radicales sont officiellement exclues du Tripos de sciences morales de Cambridge tant que celui-ci reste un monopole anglican jusqu'en 1871. Mais l'Église établie ne peut empêcher son œuvre de représenter aux yeux du monde la science morale anglaise. 

des questionnements facilitent l'émergence d'une république intellectuelle des sciences morales à l'échelle européenne, il reste que celle-ci est fondamentalement inégalitaire. Aux cas de l'Espagne et de l'Italie, traductrices massives de travaux français et anglais et faiblement exportatrices, on doit ainsi opposer l'Angleterre et la France où la balance commerciale des idées est fortement bénéficiaire, et donner un statut particulier aux petits pays bilingues comme la Suisse et la Belgique ${ }^{22}$. Ce schéma général doit être nuancé. Sur cinquante membres associés de la classe des lettres et sciences morales de l'Académie de Belgique, par exemple, on ne compte dans l'Annuaire de 1848 que 3 britanniques contre 18 français (la proportion est de 12 contre 10 dans la classe des sciences). d'Adolphe Quételet lui-même (qui cumule les positions), appartenir aux deux sections à la fois : bel exemple du caractère toujours contesté et contestable de la classification des idées et des hommes. L'Allemagne est quant à elle une puissance montante dont l'influence se fait de plus en plus sentir au cours de la deuxième moitié du XIX siècle, même si elle n'appartient pas véritablement à cet espace des sciences morales. En effet, le terme Geisteswissenschaften (transcription du terme anglais moral science tel qu'il est utilisé par John Stuart Mill), n'y est introduit qu'en 1883 par Wilhelm Dilthey qui s'inscrit alors dans un débat interne aux sciences sociales universitaires bien distinct de la problématique de Condorcet, Guizot et Whewell.

Ce n'est que dans le dernier quart du siècle que l'émergence d'un nouveau réseau de revues universitaires souvent vouées à une discipline en particulier, telles Mind (1876), la Revue historique (1876), le Quarterly Journal of Economics (1886), le Giornale degli economisti (1886), l'Economic Journal (1890) ou la Revue de métaphysique et de morale (1893) redéfiniront profondément les canaux de circulation des idées, vidant de leur dimension internationale les sciences morales et politiques. À partir de ce moment, les disciplines, désormais dotées d'une existence distincte au sein des universités, organisent la circulation internationale des œuvres.

Épilogue

18 Les sciences humaines, on le sait, sont une invention récente. Leur champ épistémologique "n'a pas été prescrit à l'avance », comme le notait Michel Foucault qui le datait du XIXe siècle ${ }^{23}$. Pourtant, on ne parle guère alors de "sciences humaines ". Les «sciences sociales" elles-mêmes, si centrales dans la plupart des travaux qui portent sur le XIX ${ }^{\mathrm{e}}$ siècle, ne sont qu'un aspect d'un champ plus vaste au sein duquel divers acteurs rivalisent pour définir et institutionnaliser les sciences de l'homme. Si l'on occulte parfois cet épisode de l'histoire des sciences humaines, c'est en partie parce que le projet qui consistait à accompagner et à différer l'essor de la démocratie est obsolète. Mais c'est peut-être aussi du fait d'une croyance trop répandue selon laquelle les sciences «non physiques » relèveraient d'une activité littéraire qui n'aurait pas de véritable tradition d'organisation institutionnelle. L'histoire des sciences morales au $\mathrm{XIX}^{\mathrm{e}}$ siècle montre qu'une telle vision est fausse. Il n'est donc pas inutile, à l'heure où se pose la question de la place des sciences humaines et sociales au sein d'un dispositif de recherche français en cours de rénovation, de se souvenir du moment européen des sciences morales, où se posa pour la première fois la question de l'organisation et de la juste représentation des différentes branches de la connaissance sur l'homme, de sa 
capacité à répondre à une demande politique et sociale, et de son insertion dans un réseau international.

\section{NOTES}

1.Plusieurs travaux récents ont contribué à renouveler notre connaissance des sciences morales en Europe. On peut mentionner en particulier, dans l'ordre chronologique, Leterrier A.-S., L'institution des sciences morales, L'Harmattan, 1995 ; Delmas C., Instituer des savoirs d'État : l'Académie des sciences morales au XIX siècle, L'Harmattan, 2006 ; Palfrey D., The Moral Science at Cambridge University, 1848-1860, thèse de doctorat non publiée, Université de Cambridge, 2001 ; Vincent J., Disestablishing Moral Science: John Neville Keynes, Religion, and the Question of Cultural Authority in Britain 1860-1900, thèse de doctorat non publiée, Université de Cambridge, 2006 ; Richard E., Sciences sociales et politiques en Espagne : autour de l'Académie royale des sciences morales et politiques (1857-1923), thèse en cours sous la direction de Vincent B., université de Paris I Panthéon-Sorbonne.

2.Pour une discussion portant sur la Grande-Bretagne voir Morell J. et Thackray A., Gentlemen of Science: Early Years of the British Association for the Advancement of Science, Oxford, Clarendon Press, 1981.

3.La lettre est reproduite au début de chaque numéro de l'Annuaire de l'Académie royale de Belgique à partir de 1846.

4.Staum M., Minerva's Message. Stabilizing the French Revolution, Montreal, Mac GillQueen's University Press, 1996.

5.Le Tripos, terme qui désigne à l'origine le tabouret à trois pieds sur lequel les étudiants s'asseyaient pour leurs examens, est synonyme de " faculté », de « cursus universitaire » ou d'» examen ».

6.Voir les travaux d'Élodie Richard, «Sciences sociales et politiques en Espagne », op. cit.

7.Pour une comparaison entre la Royal Society et l'Académie des sciences voir Brioist P., « The Royal Society and the Académie des sciences in the first half of the eighteenth century » in Charle C., Vincent J. et Winter J. (eds.), Anglo-French Attitudes: Comparisons and Transfers between English and French Intellectuals since the Eighteenth Century, Manchester, Manchester University Press, 2007, pp. 63-77.

8.Goldman L., Science, Reform, and Politics in Victorian Britain: The Social Science Association 1857-1886, Cambridge, Cambridge University Press, 2002.

9.Lavalleye J., L'Académie royale des sciences, des lettres et des Beaux-Arts de Belgique 1772-1972, Bruxelles, Palais des Académies, 1973, pp. 58-59.

10.Sur les diverses formes de l'autorité culturelle voir Charle C., Les intellectuels en Europe au XIXe siècle : essai d'histoire comparée, Seuil, 2001.

11.C'est l'expression qu'emploie Stanislas Bormans dans sa notice nécrologique sur Émile de Laveleye : Annuaire de l'Académie royale de Belgique, Bruxelles, 1895, p. 221. 12.Pour une analyse récente qui se concentre sur le cas particulier de l'économie politique, voir Stedman Jones G., La fin de la pauvreté ? Un débat historique, Paris, éditions èंe, 2007. 
13. Coleridge S.T., On the Constitution of Church and State, 1829 ; Lechevalier J., Archives des sciences morales et politiques, janvier 1834.

14.Memorias de la Real Academia de Ciencias morales y politicas, t. 1, Madrid, Imprenta Nacional, 1861, p. 21, 16-17, cité par l'Académicien belge Lallemand L., Les Grands problèmes sociaux à l'Académie royale des sciences morales et politiques d'Espagne, Paris, Picard, 1889, p. 5.

15.Rosanvallon P., Le moment Guizot, Gallimard, 1985.

16.Vincent J., Disestablishing Moral Science, op. cit.

17.Sur le succès de la « méthode comparative » voir Collini S., Burrow J. et Winch D., That Noble Science of Politics: A Study in Nineteenth-Century Intellectual History, Cambridge, Cambridge U.P., 1984.

18.Leterrier S.-A., « Un réseau de pensée européen : l'Académie des sciences morales et politique sous la Monarchie de Juillet », in Revue d'histoire du XIX ${ }^{e}$ siècle, VII (1991), pp. 37-54.

19. Mémoires de l'Académie royale des sciences morales et politiques, tome 1 consacré aux « Savants étrangers », Paris, Didot, 1841.

20.Lallemand L., Les Grands problèmes sociaux, op. cit., p. 31.

21.Leclère L., « Sciences philosophiques » in L'Académie royale de Belgique depuis sa fondation (1772-1922), Bruxelles, M. Lamertin \& M. Hayez, 1922, p. 238.

22.Pour une discussion portant sur le cas particulier de l'économie politique voir Vincent J., 'The Commerce of Ideas: Protectionism vs. Free Trade in the International Market of Economic Ideas in Britain and France circa 1900', in Anglo-French Attitudes, op. cit., pp.

194-213.

23.Foucault M., Les mots et les choses : une archéologie des sciences humaines, Gallimard, 1966, p. 355.

\section{RÉSUMÉS}

$\mathrm{Au} \mathrm{XIX}^{\mathrm{e}}$ siècle les « sciences morales » sont un mot d'ordre à la fois scientifique et politique pour toute l'Europe. À une époque où l'idée de sciences « humaines » ou " sociales » ne s'est pas encore imposée, elles sont l'expression d'un ambitieux projet pour organiser toutes les sciences «non physiques » et pour les mettre au service du progrès humain. Julien Vincent éclaire ce chapitre oublié de l'histoire des institutions scientifiques.

In the XIX ${ }^{\text {th }}$ century "moral science" was a slogan for both science and politics throughout Europe. At a time when the idea of a "human" or a "social" science was still contested, moral science was the expression of an ambitious project to organise all "non-physical" sciences and foster human progress. Julien Vincent sheds light on this forgotten chapter in the history of scientific institutions.

\section{INDEX}

Mots-clés : Europe, sciences morales, sciences politiques 


\section{AUTEUR}

\section{JULIEN VINCENT}

Julien Vincent, agrégé d'histoire et docteur en histoire contemporaine, est ATER à l'Université de Reims-Champagne-Ardennes. Il est l'auteur d'une thèse sur les sciences morales en Angleterre soutenue à Cambridge en 2006 sous la direction de Christophe Charle et de Gareth Stedman Jones. 